\title{
STOMACH
}

\section{Primary prevention of diclofenac associated ulcers and dyspepsia by omeprazole or triple therapy in Helicobacter pylori positive patients: a randomised, double blind, placebo controlled, clinical trial}

\author{
J Labenz, A L Blum, W W Bolten, B Dragosics, W Rösch, M Stolte, H R Koelz
}

Gut 2002;51:329-335

See end of article for authors' affiliations

Correspondence to:

Professor H R Koelz,

Division of

Gastroenterology,

Department of Internal

Medicine, Triemli Hospital,

Birmensdorferstrasse 497,

$\mathrm{CH}-8063$ Zurich,

Switzerland;

hrkoelz@hin.ch

Accepted for publication 9 January 2002

\begin{abstract}
Background: There is much controversy as to whether or not treatment of Helicobacter pylori reduces the occurrence of peptic ulcers during therapy with a non-steroidal anti-inflammatory drug (NSAID). Aim: To assess the efficacy of triple therapy or omeprazole on the occurrence of diclofenac associated ulcers in $\mathrm{H}$ pylori positive patients.

Methods: This was a randomised, double blind, placebo controlled, multicentre trial in $H$ pylori positive patients requiring NSAID therapy who had no past or current peptic ulcer. They received diclofenac $50 \mathrm{mg}$ twice daily for five weeks in combination with one of the four randomly assigned treatments: anti-H pylori treatment for one week (omeprazole $20 \mathrm{mg}+$ clarithromycin 500 mg+amoxicillin $1 \mathrm{~g}$, all twice daily) followed by placebo for four weeks (OAC-P); anti-H pylori treatment for one week followed by antisecretory treatment with omeprazole $20 \mathrm{mg}$ once daily for four weeks (OAC-O); omeprazole $20 \mathrm{mg}$ once daily for five weeks (O-O); or placebo for five weeks (P-P). Patients were endoscoped before and after treatment.

Results: Data from 660 patients were included in an intention to treat analysis. The occurrence of peptic ulcers in the four treatment groups during the study period was: $1.2 \%$ for OAC-P, $1.2 \%$ for OAC-O, $0 \%$ for $\mathrm{O}-\mathrm{O}$, and $5.8 \%$ for P-P ( $<<0.05$ between placebo and all active treatment groups). Patients who received active treatment developed therapy requiring dyspeptic symptoms less frequently than those who received placebo ( $p<0.05$ between placebo and all active treatment groups).

Conclusions: In $\mathrm{H}$ pylori infected patients, all three active therapies reduced the occurrence of NSAID associated peptic ulcer and dyspeptic symptoms requiring therapy.
\end{abstract}

$\mathrm{T}$ reatment with non-selective non-steroidal antiinflammatory drugs (NSAIDs) is a major cause of so-called NSAID gastropathy, including superficial mucosal damage (erosions), gastroduodenal ulcers, ulcer complications, and dyspepsia. ${ }^{12}$ As Helicobacter pylori infection can also lead to ulcers, its presence may increase the ulcer risk of NSAIDs. On the other hand, it is conceivable that the combined effects of $H$ pylori gastritis and NSAIDs actually reduces the risk of mucosal damage as $H$ pylori infection increases the synthesis of prostaglandins in the gastric mucosa by inducing cyclooxygenase $2,{ }^{3}{ }^{4}$ which may protect from drug injury, and NSAIDs may attenuate $H$ pylori induced reactive oxygen metabolite production ${ }^{5}$ thus reducing the damage otherwise caused by $H$ pylori.

Highly selective inhibitors of cyclooxygenase 2 (COX-2) are associated with a lower incidence of ulcers than conventional non-selective NSAIDs. ${ }^{6-8}$ However, while some studies indicate an ulcer incidence similar to placebo, ${ }^{9-11}$ others report higher rates than with placebo, ${ }^{12}$ in particular in $H$ pylori positive patients. ${ }^{6}$ Highly selective COX-2 inhibitors still cause dyspepsia, ${ }^{12-14}$ and simultaneous treatment with low dose aspirin appears to completely abolish the gastrointestinal advantages of highly selective COX-2 inhibitors. ${ }^{13}$ In addition, experience with the new highly selective COX-2 inhibitors is still limited, and COX-2 inhibitors have recently been accused of increasing the risk of cardiovascular events. ${ }^{15}$ Importantly, drug costs are considerably higher than those of conventional NSAIDs. Therefore, conventional NSAIDs should be prescribed for most patients not judged to be at high risk from adverse events for many years to come.
Gastric acid plays a permissive role both in $H$ pylori and NSAID induced ulcers. ${ }^{16}$ Thus proton pump inhibitors (PPI) are useful for primary and secondary ulcer prevention. ${ }^{17}$ Unfortunately, studies aimed at providing NSAID ulcer prophylaxis by treating $H$ pylori have been controversial..$^{19}$ Against this background, we initiated a large placebo controlled, double blind, four arm study in patients treated with diclofenac, to assess which of three active treatment regimens (omeprazole alone, $H$ pylori treatment alone, or the combination) is capable of reducing the incidence of gastroduodenal erosions, ulcers, and bothersome dyspepsia.

\section{METHODS}

\section{Study design}

This randomised, double blind, placebo controlled study with four parallel groups was conducted in 73 primary and secondary centres in Germany (64 centres), Austria (seven centres), and the Czech Republic (two centres) between March 1998 and August 1999, in accordance with the principles of good clinical practice and the revised Declaration of Helsinki. The study protocol, patient information, and consent form were approved by an independent ethics committees at each of the

Abbreviations: NSAID, non-steroidal anti-inflammatory drug; COX-2, cyclooxygenase 2; RR, relative risk; OAC-P, omeprazole+amoxicillin+clarithromycin, followed by placebo; OAC-O,

omeprazole+amoxicillin+clarithromycin, followed by omeprazole; O-O, omeprazole followed by omeprazole; P-P, placebo followed by placebo; $\mathrm{PPI}$, proton pump inhibitor. 
study centres, and written informed consent was obtained from each patient prior to enrolment.

\section{Selection of patients}

Patients were aged over 18 years with inflammatory or degenerative disease of the musculoskeletal system requiring treatment with an NSAID for at least five weeks, and $H$ pylori positive. Exclusion criteria were: ulcer history or an ulcer at admission endoscopy; clotting disorders; prior regular use of NSAIDs (exception was aspirin at a dose of $\leqslant 100 \mathrm{mg} /$ day), antibiotics, PPIs, misoprostol, or bismuth salts within the four weeks preceding initiation of the study; regular use of $\mathrm{H}_{2}$ receptor antagonists, prokinetics or sucralfate; systemic corticosteroids (dose corresponding to $>10 \mathrm{mg}$ prednisolone); known or suspected intolerance to a study drug; severe concomitant diseases; previous gastric surgery; pregnancy or nursing; and therapy requiring dyspepsia at admission.

\section{Study protocol}

Patients were randomised to one of four groups: omeprazole $20 \mathrm{mg}$ twice daily, amoxicillin $\mathrm{l} \mathrm{g}$ twice daily, and clarithromycin $500 \mathrm{mg}$ twice daily (OAC) for one week, followed by a four week period of treatment with placebo once daily (OAC-P); OAC for one week followed by four weeks of treatment with omeprazole $20 \mathrm{mg}$ once daily (OAC-O); omeprazole $20 \mathrm{mg}$ once daily for one plus four weeks (O-O); or placebo for one plus four weeks (P-P). Randomisation of these treatments to consecutive patient numbers was done in proportions of 1:1:1:1 within blocks of four by computer using a validated algorithm. Each centre received entire blocks to be used sequentially. Initial NSAID treatment consisted of diclofenac $50 \mathrm{mg}$ twice daily. If needed, the dose could be increased to $50 \mathrm{mg}$ three times daily during the study, and tramadol $100 \mathrm{mg}$ twice daily could be added. If therapy requiring dyspeptic complaints arose during the course of the study, the patient was initially given an antacid (Maaloxan; Rhone-Poulenc Rorer, Cologne, Germany) which was taken between meals as required, but independently of the other study medications. Appointments with the study physician were scheduled for one and five weeks after study initiation.

\section{Assessments}

Patients first underwent a rapid whole blood test for Helicobacter pylori (BM Test Helicobacter pylori; Boehringer Mannheim, Mannheim, Germany).$^{20}$ In serologically positive patients a global question on dyspeptic complaints and on musculoskeletal pain was asked and scored on a visual analogue scale, and endoscopy was performed. One antral and one corpus biopsy was investigated using the a rapid urease test (HUT; Astra GmbH, Wedel, Germany ${ }^{21}$ ). H pylori infection was assumed when, within six hours, a definitive change in colour from yellow to red occurred. Two biopsies from the antrum and corpus were assessed semiquantitatively for density of $H$ pylori and severity of gastritis, in accordance with the Houston modification of the Sydney system. ${ }^{22}$ Patients with a positive urease test were admitted to the central randomisation process, but if histology did not confirm $H$ pylori gastritis, the patient was removed from the study at the one week visit. Patients were classified as "smokers" if they smoked daily.

At baseline as well as at the one and five week visits, patients were questioned about dyspeptic complaints. Dyspepsia was graded as none, mild (not requiring therapy), and severe (requiring therapy). In addition, patients completed a $100 \mathrm{~mm}$ visual analogue scale assessment of their dyspeptic symptoms, general state of health, and pain in the musculoskeletal system. At the one and five week visits, they were also asked about signs of gastrointestinal bleeding and adverse events. Unused study medication for week 1 and for the following four week period was returned, and compliance was

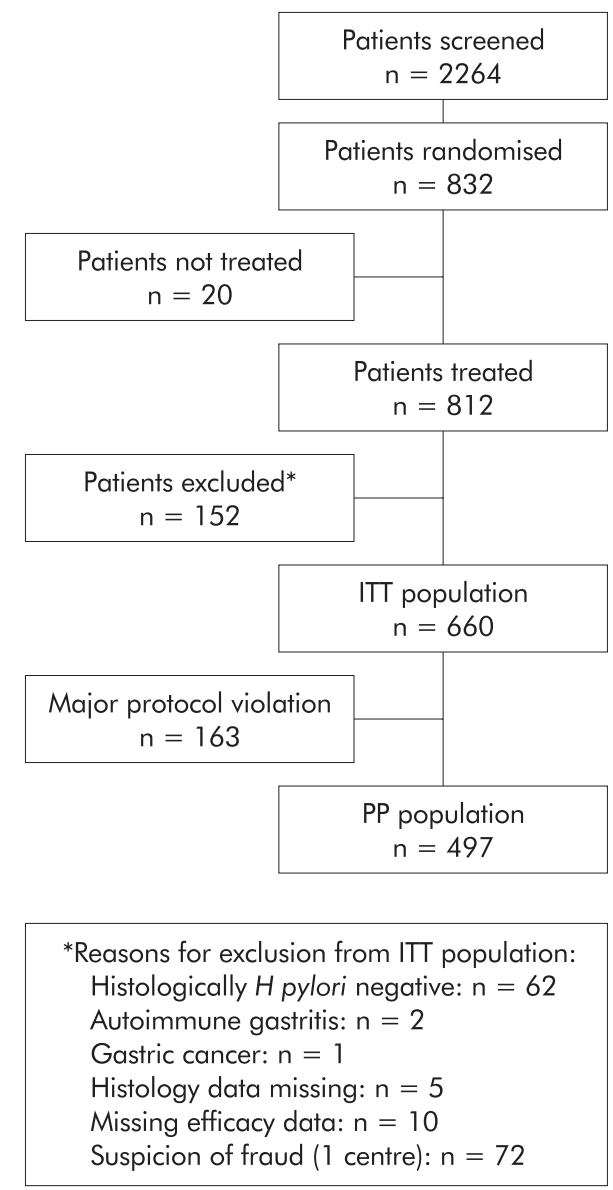

Figure 1 Numbers of patients enrolled in the study and analysed according to the intention to treat (ITT) and per protocol (PP) approaches.

checked by counting the tablets. At the five week visit, patients underwent endoscopy of the upper digestive tract, including biopsy.

Unscheduled visits were encouraged when the antiphlogistic or analgesic treatment was ineffective, or when therapy requiring dyspeptic complaints or adverse events occurred. An unscheduled endoscopy was carried out in the event of an inadequate effect of the antacid treatment or for an adverse event such as bleeding.

At every endoscopic examination, the number of erosions and ulcers (including complications), as well as the Lanza score, were recorded separately for the stomach and duodenum. An ulcer was defined as a mucosal break with a diameter $>3 \mathrm{~mm}$, identifiable by apparent depth and an inability to lift the mucosa with the biopsy forceps. In the event of an ulcer being found, additional biopsies were taken from the base and margin of the lesion.

A patient was classified as $H$ pylori negative at final endoscopy when the biopsy specimens obtained from the antrum and corpus revealed no signs of $H$ pylori infection, either in the urease test or in the histological work up. Determination of the eradication rate achieved with the various treatment regimens was not an objective of the study but is reported. However, false negative test results are likely during acid suppressive treatment in the OAC-O and O-O groups.

\section{Blinding}

The randomised treatment was given in a double blind, double dummy manner using matching placebo preparations. Active medications and corresponding placebos were similar in appearance and taste. The treatment code was broken after 


\begin{tabular}{|c|c|c|c|c|}
\hline Characteristic & $\begin{array}{l}\text { OAC-P } \\
(n=161)\end{array}$ & $\begin{array}{l}\text { OAC-O } \\
(n=173)\end{array}$ & $\begin{array}{l}O-O \\
(n=155)\end{array}$ & $\begin{array}{l}\begin{array}{l}P-P \\
(n=171)\end{array}\end{array}$ \\
\hline Age (y) (mean (SD)) & $54(14)$ & $55(14)$ & $55(13)$ & 55 (14) \\
\hline Female sex $(\%)$ & 62 & 64 & 59 & 62 \\
\hline Weight (kg) (mean (SD)) & 75 (14) & $76(15)$ & 77 (14) & $76(13)$ \\
\hline Smokers (\%) & 15 & 10 & 18 & 18 \\
\hline \multicolumn{5}{|l|}{ Disease requiring NSAIDs (\%) } \\
\hline Systemic inflammatory diseases & 24 & 24 & 27 & 22 \\
\hline Non-inflammatory diseases & 73 & 73 & 71 & 74 \\
\hline Other diseases & 4 & 3 & 2 & 4 \\
\hline Mild dyspepsia at study entry (\%) & 44 & 42 & 43 & 41 \\
\hline \multicolumn{5}{|l|}{ Lanza score " 0 " at study entry (\%) } \\
\hline Stomach & 70 & 72 & 63 & 68 \\
\hline Duodenum & 89 & 90 & 87 & 88 \\
\hline
\end{tabular}

clean filing and allocation of individual patients to intention to treat and per protocol analyses.

\section{Data management and statistical analysis}

Data were transferred to and analysed by an independent statistical institute (Institute for Numerical Statistics, Cologne, Germany). All analyses were based on SAS (version 6.11) and SPSS (version 7.5) for Windows.

The primary outcome criterion was the proportion of patients with endoscopically proved peptic ulcer(s) in any one of the three active treatment groups compared with placebo. The sample size calculation for this study was based on the assumption that about $20 \%$ of patients treated with NSAIDs develop an ulcer (primary objective), and that optimal prophylaxis, for example with omeprazole, can reduce the occurrence to less than 7.5\%. ${ }^{23}{ }^{24}$ Using a two tailed test (Fisher's exact test), a significance level of $5 \%$, and a power of $80 \%$, the study required 134 patients in each treatment arm. As the appropriateness of corrections for multiple comparisons (Bonferroni) has recently been questioned, ${ }^{25}$ we calculated the statistical significance of the major outcome variable both with and without the classical Bonferroni adjustment and also with a less conservative sharper Bonferroni procedure. ${ }^{26}$

The intention to treat analysis included all randomised patients who had taken at least one dose of the study medication and in whom $H$ pylori infection was confirmed histologically. During the study, all centres were closely monitored and one centre that had recruited 72 patients failed to pass the final audit. As fraud was suspected, and later confirmed by DNA fingerprinting of gastric biopsies, these patients were excluded from all analyses. All patients in major violation of the protocol (violation of inclusion criteria, non-compliance with study medication, major delay of scheduled visits, early termination of study) were excluded from the per protocol analysis but the safety analysis included all patients who had taken at least one dose of the study medication.

In an exploratory analysis, factors which might be associated with an ulcer or therapy requiring dyspeptic symptoms during NSAID treatment were evaluated, and the relative risk (RR) and associated 95\% confidence interval (CI) were determined. The factors explored were: active treatment ( $v$ placebo), age, sex, body weight, smoking, type of rheumatic disorder, concomitant disease, previous dyspeptic complaints, dosage of diclofenac during the study, ratio randomised/ screened patients, Lanza score at study entry, and erosions in the duodenum at study entry. With respect to the primary objective of the study, we also performed a multiple logistic regression analysis. A two sided Fisher's exact test was used to compare the proportion of patients with a given binary outcome between the treatment groups. Two sided Wilcoxon signed rank tests were used to compare ordered categorical data within the treatment groups. Finally, we calculated the relative risk reduction, absolute risk reduction, and the number needed to treat (with the corresponding 95\% CIs) with respect to ulcer and/or therapy requiring dyspepsia. ${ }^{27}$

\section{RESULTS}

\section{Patient population}

We screened a total of 2264 patients, of whom 832 were randomised to the study. Twenty patients were not treated and hence 812 patients entered the safety analysis. In addition to the 72 patients excluded from the fraudulent centre, 80 patients were excluded from the intention to treat analysis and a further 163 patients from the per protocol analysis (fig 1). No differences were noted between the treatment arms in terms of protocol violations. The demographic and clinical data of the study patients were comparable in all treatment groups (table 1). Overall, 35\% of all study patients (with equal distribution among treatment groups) said that they had suffered from NSAID associated dyspepsia in the past.

\section{Compliance}

Compliance was excellent, as judged by counting returned medication. For antibiotics or their respective placebos, $99-100 \%$ of patients in the four treatment groups were considered to be fully compliant - that is, they took at least $80 \%$ of the prescribed medication. The results were similar for diclofenac and omeprazole (or its placebo). The number of patients who did not have a second endoscopy was low ( six in the OAC-P, nine in the OAC-O, four in the O-O, and nine in the P-P group).

\section{Peptic ulcers}

Overall, 14 patients in the intention to treat population developed peptic ulcers $(>3 \mathrm{~mm})$ during treatment $(2,0,0$, and 7 patients with duodenal ulcer as well as $0,2,0$, and 3 patients with gastric ulcer in the OAC, OAC-O, O-O, and P-P groups, respectively). The rate was significantly higher in the placebo group than in all of the active treatment arms but there was no difference between the active treatments (fig 2). Using a modified Bonferroni procedure for adjustment of $\mathrm{p}$ in multiple comparisons, ${ }^{26}$ all differences remained significant. Using a conventional Bonferroni adjustment of the p level $(0.017$ for three comparisons), only the difference between the placebo and $\mathrm{O}-\mathrm{O}$ groups was significant. As all patients with ulcers qualified for the per protocol evaluation, this analysis showed similar results (not shown). Thus depending on the treatment, 

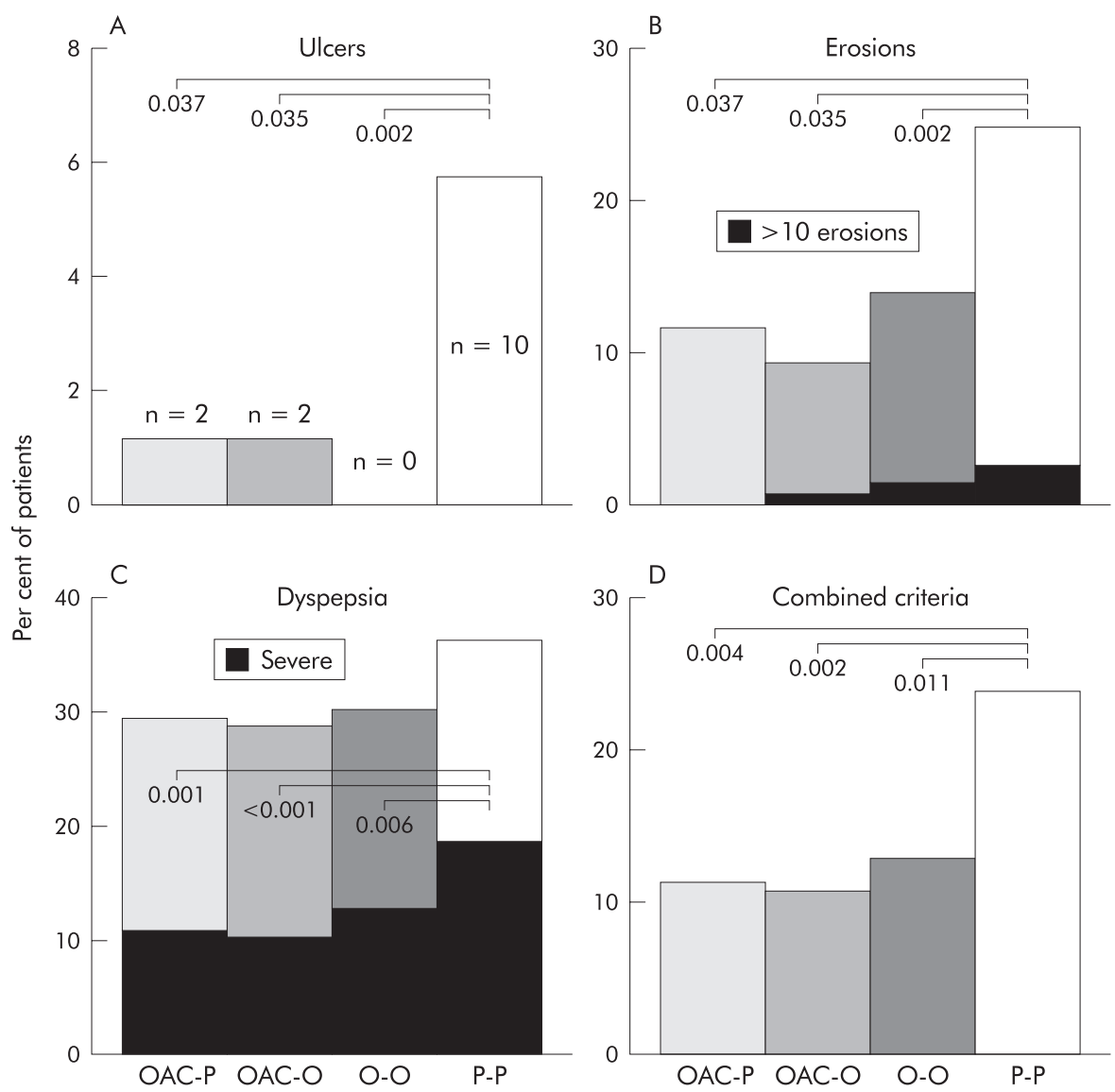

Figure 2 Incidence of ulcers (A), erosions (B), dyspeptic complaints requiring therapy (C), and combined outcome criteria (D) consisting of ulcer or $>10$ erosions or dyspepsia requiring therapy in the intention to treat population (last value during diclofenac treatment). OAC-P, omeprazole+amoxicillin+clarithromycin, followed by placebo; OAC-O, omeprazole+amoxicillin+clarithromycin, followed by omeprazole; O-O, omeprazole followed by omeprazole; P-P, placebo followed by placebo.

between 17 and 22 patients needed to be treated to prevent one ulcer (table 2). When an ulcer was defined as a lesion of $\geqslant 5 \mathrm{~mm}$, the number of ulcers in each group was as follows: $\mathrm{P}-\mathrm{P}, \mathrm{n}=9 ; \mathrm{O}-\mathrm{O}, \mathrm{n}=0(\mathrm{p} v \mathrm{P}-\mathrm{P}=0.004) ; \mathrm{OAC}-\mathrm{O}, \mathrm{n}=1(\mathrm{p}=0.01)$; and OAC-P, $\mathrm{n}=1(\mathrm{p}=0.020)$.

All ulcer patients in the placebo group remained $H$ pylori positive at the follow up endoscopy. All ulcers in the active treatment groups developed in patients who had become $H$ pylori negative. Seven of the 10 patients on placebo who developed an ulcer had therapy requiring dyspepsia during the treatment period while patients who developed ulcers during active treatment were asymptomatic. No ulcer related complications were observed.

\section{Erosions and Lanza scores}

At study entry, $24 \%$ of patients had erosions in the stomach or duodenum, with no significant differences between the treatment arms. In all of the active treatment groups, but not in the placebo group, the proportion of patients with erosions decreased significantly by the end of the study (fig 2). Comparable results were found for the Lanza score, with highly significant $(\mathrm{p}<0.001)$ differences between all active treatments and placebo.

\section{Dyspeptic symptoms}

Mild dyspeptic symptoms not requiring treatment were common prior to the start of the study (table 1). During the study,

Table 2 Relative risk reduction (RRR), absolute risk reduction (ARR), and number needed to treat (NNT) with respect to ulcer and/or therapy requiring dyspepsia

\begin{tabular}{|c|c|c|c|}
\hline & $\operatorname{RRR}(\%)(95 \% \mathrm{Cl})$ & $\operatorname{ARR}(\%)(95 \% \mathrm{Cl})$ & NNT (n) $(95 \% \mathrm{Cl})$ \\
\hline \multicolumn{4}{|l|}{ Ulcer } \\
\hline OAC-P & $79(4.5-95)$ & $4.6(0.7-8.5)$ & $22(12-143)$ \\
\hline OAC-O & $80(11.1-96)$ & $4.7(0.8-8.6)$ & $21(12-125)$ \\
\hline O-O & 100 & $5.8(2.1-9.5)$ & $17(11-48)$ \\
\hline \multicolumn{4}{|c|}{ Therapy requiring dyspepsia } \\
\hline OAC-P & $47(8.8-69)$ & $9.3(1.7-17)$ & $11(6-59)$ \\
\hline OAC-O & $48(11-69)$ & $9.5(2.0-17)$ & $11(6-50)$ \\
\hline O-O & $38(-3.5-63)$ & $7.6(-0.3-16)$ & $13(6-\infty)$ \\
\hline
\end{tabular}

OAC-P, one week treatment with omeprazole, amoxicillin, and clarithromycin, followed by four weeks of placebo; OAC-O, one week treatment with omeprazole, amoxicillin, and clarithromycin, followed by four weeks of omeprazole; O-O, five week treatment with omeprazole. 

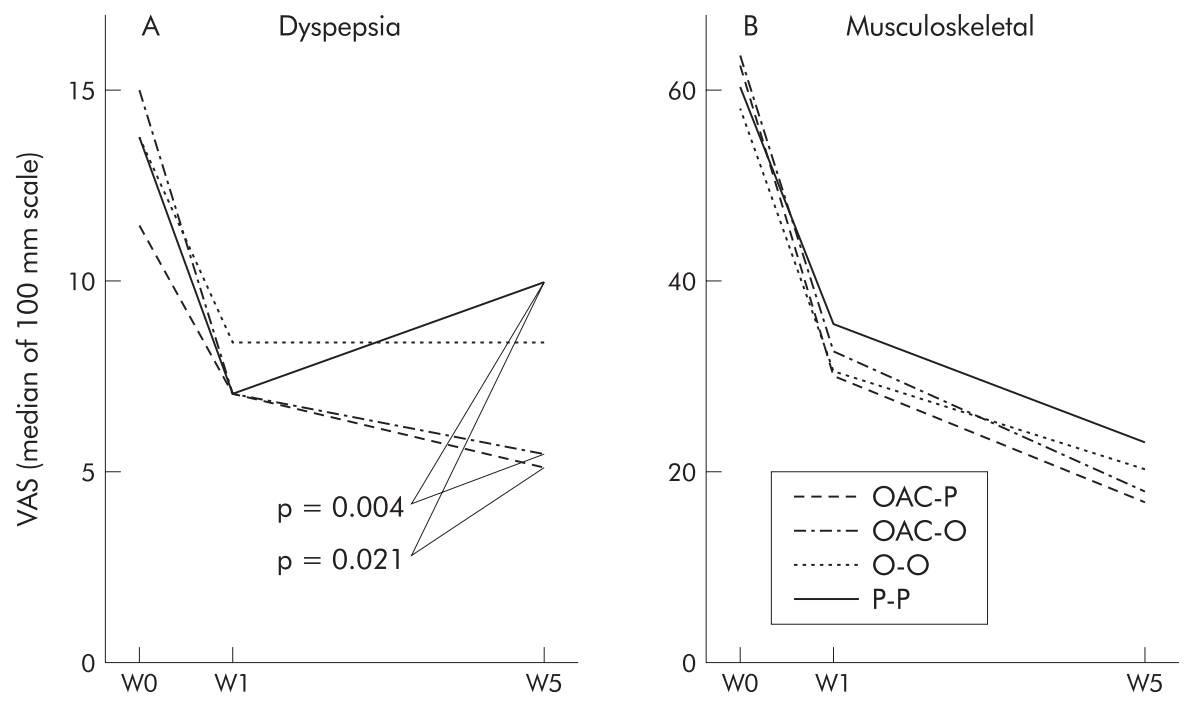

Figure 3 Development of dyspepsia (A) and musculoskeletal pain (B) during the study, as assessed by the patients on a $100 \mathrm{~mm}$ visual analogue scale. OAC-P, amoxicillin+omeprazole+clarithromycin, followed by placebo; OAC-O, amoxicillin+omeprazole+clarithromycin, followed by omeprazole; O-O, omeprazole followed by omeprazole; P-P, placebo followed by placebo.

$10.6 \%$ of patients in the OAC-P group, $10.4 \%$ of those in the OAC-O group, $12.3 \%$ of patients in the omeprazole group, and $19.9 \%$ of those in the placebo group developed therapy requiring dyspeptic symptoms (fig 2). In the placebo group, the probability of developing therapy requiring dyspeptic symptoms during treatment with diclofenac was greater in patients who, at the start of the study, already presented with mild dyspeptic complaints than in those initially symptom free $(27.1 \% \vee 14.9 \% ; \mathrm{p}=0.05)$. In the active treatment arms, mild dyspeptic complaints at the start of the study had no influence on the subsequent development of therapy requiring dyspepsia. Dyspeptic complaints, as assessed by a VAS, improved in all treatment groups. However, the OAC containing regimens were more effective than placebo (fig 3).

\section{Combined criteria}

The probability of developing an event defined as an ulcer, more than 10 erosions, or the development of therapy requiring dyspeptic symptoms ${ }^{28}$ was comparable in all of the active treatment arms, and was significantly lower than in the placebo arm (fig 2).

\section{Prognostic factors}

In the univariate analysis, the sole prognostic factor with regard to the development of a peptic ulcer was active versus placebo treatment (RR 0.14, 95\% CI 0.04-0.44; p<0.001). Active treatment was also the only prognostic factor identified by multiple logistic regression analysis. Ulcers occurred in a similar proportion of patients with $(1 / 56 ; 1.8 \%)$ and without $(13 / 590 ; 2.2 \%)$ duodenal erosion(s) at the beginning of the study. With regard to the occurrence of therapy requiring dyspeptic symptoms during the study, significant prognostic factors were assignment to active prophylactic treatment (RR $0.56,95 \%$ CI $0.38-0.82 ; \mathrm{p}=0.006$ ) and smoking (RR $1.61,95 \%$ CI $1.07-2.43 ; \mathrm{p}=0.028)$.

\section{$H$ pylori status at the end of the study}

A negative $H$ pylori status was demonstrated in $81.3 \%$ of patients in the OAC-P group, in $85.3 \%$ of patients in the OAC-O group, in $21.9 \%$ of patients in the O-O group, and in $11.8 \%$ of patients in the P-P group. Both OAC treatment groups differed significantly from the placebo (P-P) and omeprazole (O-O) groups $(\mathrm{p}<0.0001)$.
Pain control and general condition

At the start of the study, musculoskeletal pain intensity and general condition, assessed on the basis of a visual analogue scale, were comparable in all groups. During the course of the five weeks of treatment, a significant improvement in pain levels and general condition for all four treatment arms was observed $(\mathrm{p}<0.001)$, with no difference between the individual groups with respect to pain control (fig 3). However, general condition was significantly better in both patient groups receiving OAC compared with placebo (fig 3). The number of patients who needed an increase in the daily dose from $100 \mathrm{mg}$ to $150 \mathrm{mg}$ of diclofenac daily, at least for part of the study, was as follows: OAC-P 16\%, OAC-O 24\%, O-O 19\%, and P-P 22\%. Differences between the groups were not statistically significant. The proportion of patients who needed additional therapy with tramadol was similar across the treatment groups: OAC-P 6.2\%; OAC-O 6.4\%; O-O 4.5\%; and P-P $7.0 \%$.

\section{Evaluation of safety}

A total of 201 patients reported 302 adverse events. The incidence of adverse events was $26 \%$ in patients treated with OAC-P, $31 \%$ in patients treated with OAC-O, $16 \%$ in patients treated with $\mathrm{O}-\mathrm{O}$, and $26 \%$ in patients treated with P-P. The most frequently reported adverse event was diarrhoea which occurred more frequently in patients treated with antibiotics (OAC-P $8.4 \%$; OAC-O 8.8\%) than in patients assigned to the $\mathrm{O}-\mathrm{O}(3.0 \%)$ and $\mathrm{P}-\mathrm{P}(3.3 \%)$ groups, respectively.

\section{DISCUSSION}

In the present study, ulcer rates in $H$ pylori positive patients receiving diclofenac for five weeks were much higher than would be expected in $H$ pylori positive patients not taking NSAIDs. ${ }^{16}$ In the group without any preventive measuresthat is, neither eradication nor omeprazole treatment-the ulcer incidence was $6 \%$. This rate is rather low, probably due to the fact that we excluded patients with a high risk of ulcer development such as a history of peptic ulcer, severe concomitant diseases, ${ }^{1}$ and old age. In addition, all patients received an antirheumatic agent with moderate ulcer risk. ${ }^{29}{ }^{30}$ Similar rates were observed in other studies where high risk patients were excluded. ${ }^{14}$ A higher ulcer risk ${ }^{18}$ during NSAID treatment has been reported from areas with a high endemic ulcer risk such as Hong Kong. ${ }^{31}$ Possible explanations for the enhanced 
mucosal toxicity of NSAIDs in patients with $H$ pylori infection are deterioration of the mucosal barrier caused by inflammation, a higher level of apoptosis in the infected gastric mucosa, and an increase in acid secretion. ${ }^{16}{ }^{32}$ In addition, $H$ pylori may prevent gastric adaptation to NSAIDs. ${ }^{33}{ }^{34}$ In contrast, prostaglandin associated mechanisms appear to play a minor, if any, role in the interaction between H pylori and NSAIDs. ${ }^{3}$

Eradication treatment and prophylactic omeprazole appear to be equally effective in the primary prevention of NSAID associated ulcers. The effectiveness of omeprazole confirms previous studies. ${ }^{23}$ In contrast, the effectiveness of $H$ pylori eradication treatment, for which we have provided convincing evidence, has to date been considered controversial on the basis of one study with an inadequate design. ${ }^{18}$ This single study of $H$ pylori treatment as a primary prophylaxis of NSAID associated ulcers had the following major shortcomings: treatment was not double blind, but "single blind", using bismuth, which blackens the stools, as opposed to our double blind, double dummy treatment. In addition, the one week $H$ pylori eradication treatment was given before starting NSAID therapy and hence study duration was different for the two treatment groups; in our study, NSAID therapy and prophylactic treatment were started simultaneously. Furthermore, various different NSAIDs were used, while in our study all patients received diclofenac. Finally, comparisons were limited to $H$ pylori eradication with no treatment; in our study, a four treatment arm design was used allowing adequate differentiation of $H$ pylori eradication and omeprazole treatment.

Studies on the effect of $H$ pylori treatment in secondary, as opposed to primary, prevention of NSAID associated ulcers have shown a different picture. In patients with previous ulcers ${ }^{19}$ or ulcer bleed, ${ }^{35}$ omeprazole maintenance was much more effective than $H$ pylori treatment. In an earlier small study, ${ }^{36}$ ulcer recurrence after omeprazole based $H$ pylori treatment was not significantly reduced compared with short term omeprazole therapy alone. The finding that the rate of ulcer recurrence of $H$ pylori positive patients on long term NSAIDs was lower than in $H$ pylori negative patients during maintenance treatment with omeprazole ${ }^{28}$ may be explained by the higher effectiveness of omeprazole on gastric acidity in $H$ pylori positive subjects ${ }^{37}$ and improvement in gastric adaptation by the suppression or elimination of the infection in the antrum under PPI treatment. ${ }^{34} 39$

The prevalence of dyspeptic symptoms at the start of the present study corresponded to that of a large general population of non-selected blood donors in Germany. ${ }^{40}$ In the placebo group, NSAID treatment caused therapy requiring dyspeptic symptoms in approximately $20 \%$ of patients (particularly in those with mild dyspeptic complaints prior to the study), a risk which could be halved both by omeprazole alone and triple therapy against $H$ pylori. The effect of $H$ pylori eradication is surprising as in population based studies, subjects with $H$ pylori infection do not demonstrate dyspeptic symptoms more frequently than non-infected patients ${ }^{41}$ and, in non-ulcer dyspepsia, treatment of $H$ pylori has no clear effect in comparison with placebo. ${ }^{42}$ This indicates that in the pathogenesis of NSAID associated dyspepsia, factors other than those operative in non-ulcer dyspepsia are involved.

In conclusion, one week of triple therapy with omeprazole, clarithromycin, and amoxicillin is as effective as co-treatment with omeprazole alone in the primary prevention of ulcers and dyspepsia during short term treatment with diclofenac in $H$ pylori infected patients of low risk. However, in view of the very low incidence of ulcers (with no complications) in the placebo group of our carefully selected population, we hesitate to recommend a general "test and treat" strategy for $H$ pylori at the beginning of short term NSAID therapy. This opinion is also in line with a recent decision analysis model regarding the clinical and economic impact of $H$ pylori screening in patients requiring chronic NSAID treatment. ${ }^{43}$

\section{ACKNOWLEDGEMENT}

We are indebted to Dr Madeline Frame for assistance with the drafting and editing of the manuscript and to all other personnel who took part in the study.

\section{APPENDIX}

In addition to the authors, other members of the TON Study Group were as follows:

Austria: R Fiedler, W Florian, E Hentschel, H Hügel, G Mathis, H Schöngut, W Weiss; Czech Republic: J Spicák, D Zavoral; Germany: A B Abrar, A Achim, I Auer, C Bauknecht, P Berressem, W Biewer, G Böhm, H Böneke, W Brandt, H Bremer, H Brinkhoff, M Buchner, R Deutscher, R Dockhorn, E Dombrowski, M Fiedler, D Gragert, I Gromnica, W Güttel, C Haase, J Habbig, H Hagel, U Hahn, M Hampel, B Hawickhorst, K Hey, J Huesmann, K Judaschke, H Juergens, A Kabbani, R Khaffaf, C Klein, M Klöters, J Kobrle, M Koch, P Kohls, K Kolbe, J Kreutzer, A Leodolter, H Lichti, D Linden, H Lindner, E Massmann, P Mayr, E Meier, U Meyer, A Moslehner, M Mucha, A Müser, J Nies, S Noack, J Pankow, P Pech, H Petzold, T Rachel, M Römhild, G Rosprich, T Schäfer, H Schleenbecker, B Schmidt, A Schober, B Schumacher, E Schütz, M Sellinger, M Suleiman, H Teichmüller, W Thies, T Tibroni, D Timm, P Tippmann, H Toluipur, H Treichel, A Vogel, S Wassenberg, G Wiest, G Wilhelms, W Wilke, D Wolff, K Ziegler.

\section{Authors' affiliations}

J Labenz, Department of Medicine, Jung-Stilling Hospital, Siegen, Germany

A L Blum, Division of Gastroenterology, Centre Hospitalier Universitaire Vaudois, Lausanne, Switzerland

W W Bolten, Rheumaklinik Wiesbaden II, Wiesbaden, Germany B Dragosics, Gesundheitszentrum Süd, Vienna, Austria

W Rösch, Department of Medicine, Hospital Nordwest, Frankfurt, Germany

M Stolte, Institute of Pathology, Klinikum Bayreuth, Bayreuth, Germany

H R Koelz, Division of Gastroenterology, Department of Internal

Medicine, Triemli Hospital, Zurich, Switzerland

\section{REFERENCES}

1 Wolfe MM, Lichtenstein DR, Singh G. Gastrointestinal toxicity of nonsteroidal antiinflammatory drugs. N Engl J Med 1999;340:1888-99.

2 NIH Consensus Development Panel on Helicobacter pylori in Peptic Ulcer Disease. Helicobacter pylori in peptic ulcer disease. JAMA 1994;272:65-9.

3 Laine L, Cominelli F, Sloane R, et al. Interaction of NSAIDs and Helicobacter pylori on gastrointestinal injury and prostaglandin production: a controlled double-blind trial. Aliment Pharmacol Ther 1995; 9:127-35.

4 McCarthy CJ, Crofford L, Greenson J, et al. Cyclooxygenase-2 expression in gastric antral mucosa before and after eradication of Helicobacter pylori infection. Am J Gastroenterol 1999;94:1218-23.

5 Jones-Blackett S, Hull MA, Davies GR, et al. Non-steroidal anti-inflammatory drugs inhibit Helicobacter pylori-induced human neutrophil reactive oxygen metabolite production in vitro. Aliment Pharmacol Ther 1999;13:1653-61.

6 Emery P, Zeidler H, Kvien TK, et al. Celecoxib versus diclofenac in long-term management of rheumatoid arthritis: randomised double-blind comparison. Lancet 1999:354:2106-11.

7 Langman MJ, Jensen DM, Watson DJ, et al. Adverse upper gastrointestinal effects of rofecoxib compared with NSAIDs. JAMA 1999;282:1929-33

8 Schnitzer TJ, Truitt K, Fleischmann R, et al. The safety profile, tolerability, and effective dose range of rofecoxib in the treatment of rheumatoid arthritis. Phase II Rofecoxib Rheumatoid Arthritis Study Group. Clin Ther 1999;21:1688-702.

9 Laine L, Harper S, Simon T, et al. A randomized trial comparing the effect of rofecoxib, a cyclooxygenase 2-specific inhibitor, with that of ibuprofen on the gastroduodenal mucosa of patients with osteoarthritis. Rofecoxib Osteoarthritis Endoscopy Study Group. Gastroenterology 1999; 117:776-83.

10 Simon LS, Weaver AL, Graham DY, et al. Anti-inflammatory and upper gastrointestinal effects of celecoxib in rheumatoid arthritis: a randomized controlled trial. JAMA 1999;282:1921-8.

11 Hawkey C, Laine L, Simon T, et al. Comparison of the effect of rofecoxib (a cyclooxygenase 2 inhibitor), ibuprofen, and placebo on the gastroduodenal mucosa of patients with osteoarthritis: a randomized, double-blind, placebo-controlled trial. The Rofecoxib Osteoarthritis Endoscopy Multinational Study Group. Arthritis Rheum 2000;43:370-7.

12 Hawkey CJ, Jackson L, Harper SE, et al. Review article: the gastrointestinal safety profile of rofecoxib, a highly selective inhibitor of cyclooxygenase-2, in humans. Aliment Pharmacol Ther 2001;15:1-9.

13 Silverstein FE, Faich G, Goldstein JL, et al. Gastrointestinal toxicity with celecoxib vs nonsteroidal anti-inflammatory drugs for osteoarthritis and rheumatoid arthritis: the CLASS study: A randomized controlled trial. Celecoxib Long-term Arthritis Safety Study. JAMA 2000;284: 1247-55. 
14 Bombardier C, Laine L, Reicin A, et al. Comparison of upper gastrointestinal toxicity of rofecoxib and naproxen in patients with rheumatoid arthritis. VIGOR Study Group. N Engl J Med 2000;343: 1520-8

15 Mukheriee D, Nissen SE, Topol EJ. Risk of cardiovascular events associated with selective COX-2 inhibitors. JAMA 2001;286:954-9.

16 Malfertheiner P, Labenz J. Does Helicobacter pylori status affect nonsteroidal anti-inflammatory drug-associated gastroduodenal pathology? Am J Med 1998;104:35-40S.

17 Hawkey CJ. Personal review: Helicobacter pylori, NSAIDs and cognitive dissonance. Aliment Pharmacol Ther 1999:13:695-702.

18 Chan FK, Sung JJ, Chung SC, et al. Randomised trial of eradication of Helicobacter pylori before non-steroidal anti-inflammatory drug therapy to prevent peptic ulcers. Lancet 1997;350:975-9.

19 Hawkey CJ, Tulassay Z, Szczepanski L, et al. Randomised controlled trial of Helicobacter pylori eradication in patients on non-steroidal anti-inflammatory drugs: HELP NSAIDs study. Helicobacter Eradication for Lesion Prevention. Lancet 1998;352:1016-21.

20 Moayyedi P, Carter AM, Catto A, et al. Validation of a rapid whole blood test for diagnosing Helicobacter pylori infection. BM 1997;314:119.

21 Labenz J, Börsch G, Peitz U, et al. Validity of a novel biopsy urease test (HUT) and a simplified ${ }^{13} \mathrm{C}$-urea breath test for diagnosis of Helicobacter pylori infection and estimation of the severity of gastritis. Digestion 1996:57:391-7.

22 Dixon MF, Genta RM, Yardley JH, et al. Classification and grading of gastritis. The updated Sydney System. International Workshop on the Histopathology of Gastritis, Houston 1994. Am J Surg Pathol 1996;20:1161-81.

23 Cullen D, Bardhan KD, Eisner M, et al. Primary gastroduodenal prophylaxis with omeprazole for non-steroidal anti-inflammatory drug users. Aliment Pharmacol Ther 1998;1 2:135-40.

24 Ekström P, Carling L, Wetterhus S, et al. Prevention of peptic ulcer and dyspeptic symptoms with omeprazole in patients receiving continuous non-steroidal anti-inflammatory drug therapy. Scand J Gastroenterol 1996;31:753-8.

25 Perneger TV. What's wrong with Bonferroni adjustments. BM 1998;316:1236-8

26 Hochberg Y. A sharper Bonferroni procedure for multiple tests of significance. Biometrika 1988:75:800-2.

27 Altman DG. Confidence intervals for the number needed to treat. $B M$ 1998;317:1309-12

28 Hawkey CJ, Karrasch JA, Szczepanski L, et al. Omeprazole compared with misoprostol for ulcers associated with nonsteroidal antiinflammatory drugs. Omeprazole versus Misoprostol for NSAID-induced Ulcer Management (OMNIUM) Study Group. N Engl J Med 1998;338:727-34

29 Garcia Rodriguez LA, Jick H. Risk of upper gastrointestinal bleeding and perforation associated with individual non-steroidal anti-inflammatory drugs. Lancet 1994;343:769-72.
30 Langman MJ, Weil J, Wainwright $P$, et al. Risks of bleeding peptic ulce associated with individual non-steroidal anti-inflammatory drugs. Lancet 1994;343: 1075-8.

31 Lam SK. Epidemiology and genetics of peptic ulcer. Gastroenterol Jpn 1993;28(suppl 5): 145-57.

32 Leung WK, To KF, Chan FK, et al. Interaction of Helicobacter pylori eradication and non-steroidal anti- inflammatory drugs on gastric epithelial apoptosis and proliferation: implications on ulcerogenesis. Aliment Pharmacol Ther 2000;14:879-85.

33 Konturek JW, Dembinski A, Konturek SJ, et al. Infection of Helicobacter pylori in gastric adaptation to continued administration of aspirin in humans. Gastroenterology 1998;114:245-55.

34 Lipscomb GR, Campbell F, Rees WD. The influence of age, gender, Helicobacter pylori and smoking on gastric mucosal adaptation to non-steroidal anti-inflammatory drugs. Aliment Pharmacol Ther 1997;1 1:907-12.

35 Chan FK, Chung SC, Suen BY, et al. Preventing recurrent upper gastrointestinal bleeding in patients with Helicobacter pylori infection who are taking low-dose aspirin or naproxen. N Engl J Med 2001;344:967-73.

36 Bianchi Porro G, Parente F, Imbesi et al. Role of Helicobacter pylori in ulcer healing and recurrence of gastric and duodenal ulcers in longterm NSAID users. Response to omeprazole dual therapy. Gut 1996:39:22-6.

37 Yeomans ND, Tulassay Z, Juhasz L, et al. A comparison of omeprazole with ranitidine for ulcers associated with nonsteroidal antiinflammatory drugs. Acid Suppression Trial: Ranitidine versus Omeprazole for NSAID-associated Ulcer Treatment (ASTRONAUT) Study Group. N Engl J Med 1998;338:719-26.

38 Labenz J, Tillenburg B, Peitz U, et al. Helicobacter pylori augments the $\mathrm{pH}$-increasing effect of omeprazole in patients with duodenal ulcer. Gastroenterology 1996;110:725-32.

39 Schenk BE, Kuipers EJ, Nelis GF, et al. Effect of Helicobacter pylori eradication on chronic gastritis during omeprazole therapy. Gut 2000;46:615-21.

40 Holtmann G, Goebell H, Holtmann M, et al. Dyspepsia in healthy blood donors. Pattern of symptoms and association with Helicobacter pylori. Dig Dis Sci 1994;39:1090-8.

41 Agreus L, Engstrand L, Svardsudd K, et al. Helicobacter pylori seropositivity among Swedish adults with and without abdominal symptoms. A population-based epidemiologic study. Scand J Gastroenterol 1995;30:752-7.

42 Blum AL, Talley NJ, O'Morain C, et al. Lack of effect of treating Helicobacter pylori infection in patients with nonulcer dyspepsia. N Engl J Med 1998;339:1875-81.

43 Scheiman JM, Bandekar RR, Chernew ME, et al. Helicobacter pylori screening for individuals requiring chronic NSAID therapy: a decision analysis. Aliment Pharmacol Ther 2001;15:63-71. 